\title{
On the formation of oxygen-neon white dwarfs in close binary systems
}

\author{
P. Gil-Pons ${ }^{1}$ and E. García-Berro ${ }^{1,2}$ \\ 1 Departament de Física Aplicada, Universitat Politècnica de Catalunya, c/Jordi Girona s/n, Módul B-4, \\ Campus Nord, 08034 Barcelona, Spain \\ e-mail: pilar@fa.upc.es \\ 2 Institute for Space Studies of Catalonia, c/Gran Capitá 2-4, Edif. Nexus 104, 08034 Barcelona, Spain
}

Received 22 February 2001 / Accepted 25 May 2001

\begin{abstract}
The evolution of a star of initial mass $10 M_{\odot}$, and metallicity $Z=0.02$ in a Close Binary System (CBS) is followed from its main sequence until an ONe degenerate remnant forms. Restrictions have been made on the characteristics of the companion as well as on the initial orbital parameters in order to avoid the occurrence of reversal mass transfer before carbon is ignited in the core. The system undergoes three mass loss episodes. The first and second ones are a consequence of a case B Roche lobe overflow. During the third mass loss episode stellar winds may play a role comparable to, or even more important than Roche lobe overflow. In this paper, we extend the previously existing calculations of stars of intermediate mass belonging to close binary systems by following carefully the carbon burning phase of the primary component. We also propose different possible outcomes for our scenario and discuss the relevance of our findings. In particular, our main result is that the resulting white dwarf component of mass 1.1 $M_{\odot}$ more likely has a core composed of oxygen and neon, surrounded by a mantle of carbonoxygen rich material. The average abundances of the oxygen-neon rich core are $X\left(\mathrm{O}^{16}\right)=0.55, X\left(\mathrm{Ne}^{20}\right)=0.28$, $X\left(\mathrm{Na}^{23}\right)=0.06$ and $X\left(\mathrm{Mg}^{24}\right)=0.05$. This result has important consequences for the Accretion Induced Collapse scenario. The average abundances of the carbon-oxygen rich mantle are $X\left(\mathrm{O}^{16}\right)=0.55$, and $X\left(\mathrm{C}^{12}\right)=0.43$. The existence of this mantle could also play a significant role in our understanding of cataclysmic variables.
\end{abstract}

Key words. stars: evolution - stars: binaries: general - stars: white dwarfs

\section{Introduction}

Intermediate mass close binaries are defined as those systems in which the primary component develops a degenerate carbon-oxygen core, after burning central helium in non-degenerate conditions. From the orbital parameters in these systems, we see that periods are small enough that the possibility of mass transfer due to Roche Lobe overflow is enabled. The evolution of low - to intermediate - mass stars belonging to close binary systems has been widely studied so far and, even though many questions still remain unsolved, important contributions have already been made on this subject. One of these questions concerns the evolution of heavy-weight intermediate mass stars (that is, primary stars with masses between $\sim 8$ and $11 M_{\odot}$ ) belonging to close binary systems. For the case of isolated stars, this range of stellar masses corresponds to stars for which, after exhaustion of central helium, carbon is ignited

Send offprint requests to: E. García-Berro, e-mail: garcia@fa.upc.es under conditions of partial degeneracy. Ultimately, these stars become Super-AGB stars with ONe cores (Ritossa et al. 1996). For the case of stars within this mass range belonging to binary systems, very few comments can be made, either because most of the calculations do not follow the evolution through the carbon burning phase or, simply, because the existing calculations focus mostly on a lower segment of masses.

For instance, Whyte \& Eggleton (1980) studied the evolution of stars of up to $3 M_{\odot}$ belonging to semidetached systems. These authors later extended their work to more general scenarios in which accretion and mass transfer between low mass contact binaries were included (Whyte \& Eggleton 1985). Van der Linden (1987) also performed conservative Case B evolutionary calculations for several masses of the primary, ranging from $3 M_{\odot}$ to $12 M_{\odot}$, but the evolution through the carbon burning phase was not followed. Besides the work they have performed in the field of massive binaries, de Loore \& Vanbeveren (1992, 1994, and references therein) have also focused on 
the evolution of intermediate mass close binary systems (de Loore \& Vanbeveren 1995). However, they were only able to follow the evolution of the primary star until the exhaustion of the helium in the core. Their calculations included both non-conservative (de Loore \& de Greve 1992) and conservative mass transfer (de Loore \& Vanbeveren 1995). Very recently, Nelson \& Eggleton (2001) have performed a very comprehensive work on intermediate mass close binary systems, exploring 5500 evolutionary tracks of mostly Case A conservative mass transfer systems. The upper mass limit in this case was $\sim 50 M_{\odot}$ but, again, in most of the cases the evolution during the carbon burning phase was not followed or it was aborted earlier (when the carbon luminosity exceeded $\left.1 L_{\odot}\right)$. In another recent work Han et al. (2000) determined the final mass relation for binary systems with the mass of the components ranging between 3 and $8 M_{\odot}$, starting mass loss at different times of the Hertzsprung-Russell gap. However, in these studies the assumption of conservative mass transfer was adopted and justified by the conditions of the case they consider which, in spite of corresponding to an important portion of the real cases, cannot account for all of them.

Iben $(1985,1991)$ has extensively reviewed the physical mechanisms relevant to binary systems and has thoroughly discussed the evolution of intermediate mass close binary systems, offering an excellent overview of their evolution until very late stages, proposing several different scenarios and providing their probabilities of occurrence. Also, Iben \& Tutukov (1984) have proposed different evolutionary scenarios for heavy-weight intermediate mass close binary systems as progenitors of SNe Ia. In spite of the fact that this mass interval contains a good fraction of the stars which are massive enough to ignite carbon in a non-explosive way, the evolution of these systems has been much neglected until very recently. The pioneering works of Miyaji et al. (1980) and Woosley et al. (1980) lead to the conclusion that the stars of this mass interval belonging to close binary systems would lose most of their mass and, moreover, would develop electron-degenerate ONe cores after the carbon burning phase. In a second phase, due to accretion from the secondary, the central density would increase until the threshold for electron capture on ${ }^{24} \mathrm{Mg}$ and ${ }^{24} \mathrm{Na}$ would be reached first, and on ${ }^{20} \mathrm{Ne}$ and ${ }^{20} \mathrm{~F}$ later. The process of electron capture on these nuclei would finally trigger the explosive ignition of neon and oxygen at very high densities $\left(\rho_{\mathrm{c}} \gtrsim 2 \times 10^{10} \mathrm{~g} \mathrm{~cm}^{-3}\right)$. At these densities, fast electron captures on the incinerated material would bring the Chandrasekhar mass below the actual mass of the ONe core and induce gravitational collapse to neutron star dimensions. Although there is a general agreement that electron-capture induced collapse could be successful, a major drawback of this scenario is that no detailed pre-collapse models existed in the literature. For instance, in most of the calculations, the evolution during the mass loss phase was not followed in full detail (Nomoto 1984) or the evolution during the carbon burning phase was oversimplified by introducing the so-called steady burning approximation
(Saio \& Nomoto 1998). Therefore, the depicted evolutionary scenario could be substantially modified due to the presence of these approximations.

Very recently, the evolution leading to the formation of white dwarfs with ONe cores in close binary systems is being reinvestigated. For instance, Domínguez et al. (1994) have studied the formation of an ONe white dwarf through mass transfer in a close binary system. On the other hand, in a series of recent papers (García-Berro \& Iben 1994; Ritossa et al. 1996; García-Berro et al. 1997; Iben et al. 1997; Ritossa et al. 1999) the evolution of isolated heavyweight intermediate mass stars has also been carefully followed from the main sequence phase up to exhaustion of carbon in the core. Perhaps one of the most important conclusions of these papers is that isolated white dwarfs with masses $M_{\mathrm{WD}} \gtrsim 1.0 M_{\odot}$ would most likely have an $\mathrm{ONe}$ core surrounded by a mantle of carbon-oxygen rich material. This bears important consequences for the abovementioned accretion-induced collapse scenario because all the existing calculations assume that the composition of the He-exhausted core is carbon-free. Nevertheless, these authors studied only the mass-conservative evolutionary tracks for the relevant range of stellar masses, whereas in a close binary system the composition of the final remnant could be dramatically affected by the previous evolutionary phase if the star is interacting with a companion. In any case, progress in the right direction has been made, but further exploration is still worthwhile.

In this paper we follow the evolution of a $10 M_{\odot}$ model star of solar metallicity belonging to a close binary system, from its main sequence phase until an oxygen-neon core develops. In order to keep consistency with our previous results, for the calculations reported in this paper we have used the same evolutionary code described in Ritossa et al. (1996). In particular we have not considered overshooting, as was done there. This procedure might lead to somewhat smaller cores than in the case in which overshooting is considered, but we expect the differences to be small. Different mass loss episodes are caused by the presence of a close companion and are followed in detail. Specifically, the model loses most of its hydrogen-rich envelope in a case B Roche-lobe overflow episode. It is worth mentioning at this point that we have taken special care in treating the mass zoning and the time steps during the mass loss episodes and the carbon burning phase (about 2000 Lagrangian mesh points are used during the most problematic phases). Moreover, the distribution of mesh points is regularly updated at each time step. Our algorithm puts mesh points where they are most needed (that is, where the gradients of the physical variables are strong) and eliminates them where they are not necessary. Once this is done, the values of the physical variables are interpolated at the new mesh points in order to properly compute the temporal derivatives. If necessary the mesh can be updated at each iteration.

As is usually done, throughout this work we will refer to the star that is initially more massive as the primary component whereas the secondary component will be its 


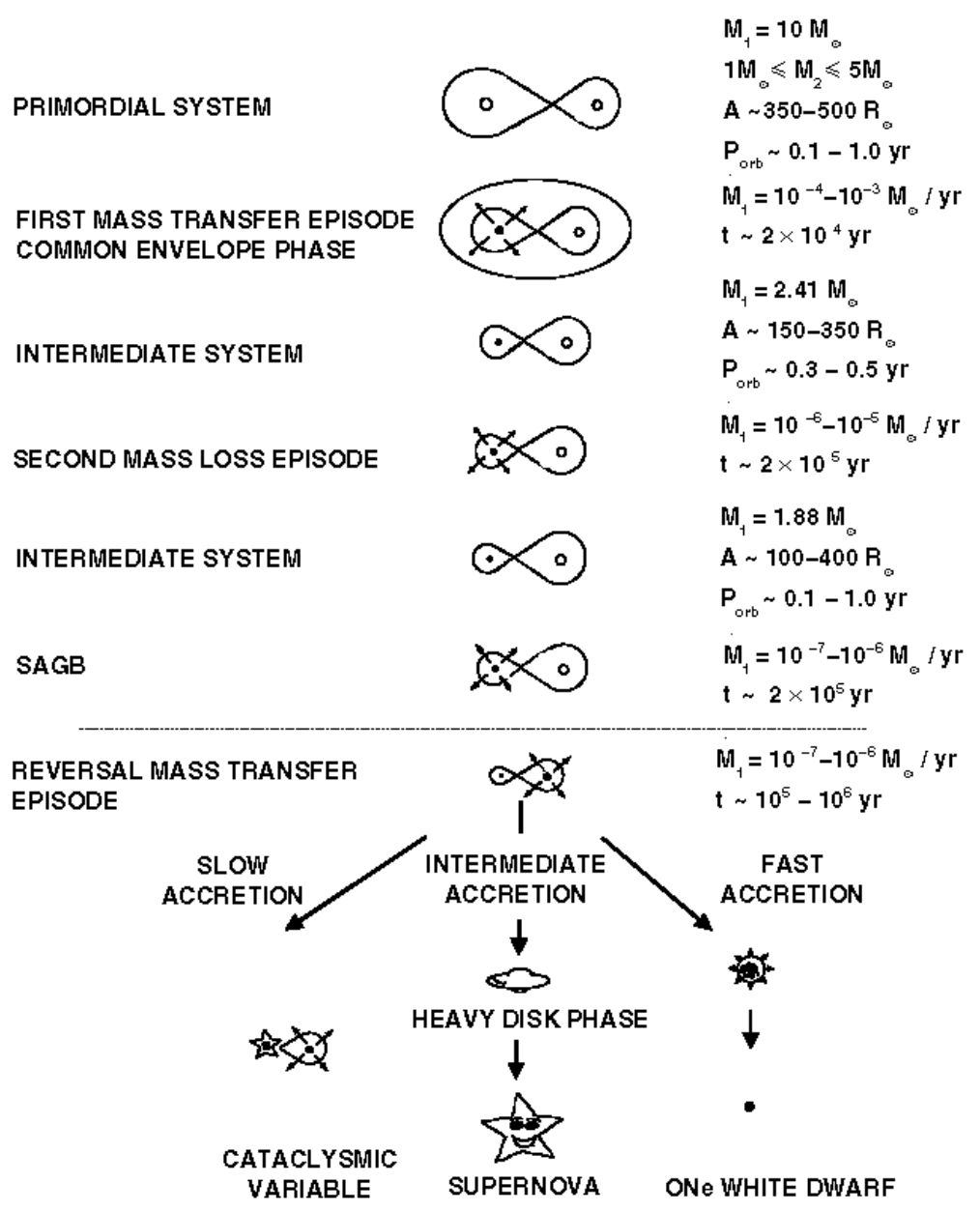

Fig. 1. Approximate evolution of the orbital parameters of the binary system and outline of the possible final outcomes. The dashed line divides the calculations reported in this paper and the three possible outcomes.

companion. We have assumed that the initial orbital parameters and the mass of the secondary component allow the whole evolution to proceed without reversal mass transfer and without disruption or merger events. This poses some constraints on the mass of the secondary which will be discussed later.

The plan of the paper is the following. In the second section of this paper we explain our choice of the initial orbital parameters and the assumed scenario. In the third section we present a description of the overall evolution of our model star until carbon ignition sets in, and we compare it with the evolution of an isolated star of the same mass. This section is also devoted to the study of the resulting mass loss episodes. In the fourth section we briefly describe the evolution during the carbon burning phase and we discuss the final characteristics of the remnant. Finally our major findings and conclusions are described in Sect. 5.

\section{The scenario}

In this section we set up our evolutionary scenario and we describe our choices for the initial orbital parameters. The reader should keep in mind that our main goal is to pro- vide a successful scenario to test the formation of massive ONe white dwarfs in close binary systems that could ultimately lead either to a cataclysmic variable or to collapse to neutron star dimensions through the accretion-induced collapse alternative. Therefore our choice of the initial parameters of the binary system is effectively influenced by the desired final outcome. A possible observational counterpart of the proposed scenario could be the binary system IK Peg (HR 8210, HD 204188), which has an orbital period of 21.7 days, and it is composed of a massive white dwarf of mass $M_{\mathrm{WD}} \simeq 1.15 M_{\odot}$ and a main sequence star of $1.4 M_{\odot}$ (Smalley et al. 1996).

Our starting point is a primordial system composed of a $10 M_{\odot}$ star and its $\lesssim 5 M_{\odot}$ companion, which we will refer to as the primary and secondary components respectively (see Fig. 1). The system undergoes a case B mass transfer, this being the most likely possibility, and so the primary starts losing mass after the onset of hydrogen burning in a shell. Unlike early case-B mass loss episodes, late case-B mass transfer has been little studied up to now. The reasons for this are multiple but perhaps the most important one is the additional computational difficulties that arise when studying such a phase. However, it has been recently pointed out (Tauris \& Dewi 2001) 
that the actual definition of the resulting core of the primary after the mass loss episode could influence the final orbital parameters. We consider it interesting, therefore, to study a late case-B mass loss episode. This determines the range of values for the initial Roche lobe radius of the primary, that we actually choose by considering the evolutionary track for the single $10 M_{\odot}$ model star followed by García-Berro et al. (1994), and we keep it constant at $210 R_{\odot}$ during the whole process (see the discussion below). Nonetheless, it is worth mentioning that we have conducted a series of numerical experiments in which the Roche lobe radius has been changed to values as small as $120 R_{\odot}$ and we have not found significant differences. Given the initial mass relation and the Roche lobe of the primary, we can obtain the orbital separation between both components by appliying the equation (Eggleton 1983):

$$
\frac{R_{\mathrm{L}}}{A}=\frac{0.46 q^{2 / 3}}{0.6 q^{2 / 3}+\ln \left(1+q^{1 / 3}\right)}
$$

where $R_{\mathrm{L}}$ denotes the effective radius of the Roche lobe of the primary, $A$ indicates the orbital separation and $q$ is the mass ratio $\left(M_{1} / M_{2}\right)$ between the components. The initial orbital separation turns out to be $A \sim 150-350 R_{\odot}$ and the orbital period is $P_{\text {orb }} \sim 0.1-1.0 \mathrm{yr}$, depending on the initial mass of the companion (between 1 and $5 M_{\odot}$ ), which are reasonable values.

As it will be shown in detail in the next section, a number of uncertainties are involved in the first mass loss episode. These uncertainties are basically caused by the formation of a common envelope. This common envelope is formed when the primary star attains the highest values for its mass loss rates, and most probably removes a large amount of mass and angular momentum from the system. Consequently, the evolution of the orbital parameters cannot be accurately followed, and this can only be done in an approximate way. In order to determine the mass lost by the primary, we assume that all the matter overflowing the Roche lobe is lost by the star, and we simply stop the process when its radius dramatically decreases below that of the Roche lobe (see Sect. 3.1).

The second mass loss episode starts when the surface radius of the primary again exceeds that of its Roche lobe. In this case, this is due to the onset of helium burning in the shell. This process is most likely conservative both in mass and in angular momentum and, again, stops when the radius of the primary decreases below the value of the Roche lobe. After this temporary decrease in radius, carbon ignition continues and the primary starts the ascent along the Super-AGB but does not fill again its Roche lobe. Once carbon is exhausted in the innermost regions, it is a stellar wind rather than Roche lobe overflow that induces mass loss and, thus, we use the parametrization of Nieuwenheuzen \& de Jager (1990) in order to account for the mass loss rates. As happened during the first mass loss episode, there are several uncertainties involved in this process, which can have consequences on the final fate of the system. In fact, depending on the choice of the efficiency of the winds, the resulting orbital parameters can vary significantly (Umeda et al. 1999). If the system survives the Super-AGB phase, we are left with an ONe white dwarf, as a result of the evolution of the primary, and a main sequence star that, at some time, will fill its Roche lobe and give rise to reversal mass transfer.

Even though we do not mean to get deep into the study of the probability of occurrence of the different final outcomes, we will briefly outline the different possibilities for the final stages of the life of the binary in terms of the mass transfer rates during the reversal mass transfer. Depending on this mass transfer, the final outcome can be either a cataclysmic variable if it is lower than a critical rate, a supernova explosion if it is larger or an ONe white dwarf if it is much larger. A more detailed study can be found in Sect. 5 .

\section{Evolution until the beginning of the carbon burning phase and main mass loss episodes}

The presence of a close companion has several consequences for the evolution of our $10 M_{\odot}$ model star. In particular, there are two Roche-lobe overflow mass loss episodes. The first one occurs just after the main sequence phase when our model star reaches red giant dimensions, and the second one happens shortly after the exhaustion of central helium.

Figure 2 shows the evolution of our model in the Hertzsprung-Russell diagram. Times to evolve to each labeled point along the evolutionary track are given in the second column of Table 1, where we also provide the most important characteristics of these particular models for the hydrogen and helium burning phases. Also shown in the last columns of Table 1 are the mass of the primary and secondary stars $\left(M_{1}\right.$ and $\left.M_{2}\right)$, and the expected orbital period $\left(P_{\text {orb }}\right)$. The solid lines represent the evolutionary phases during which no mass loss occurs, whereas the dotted lines correspond to the evolutionary phases where mass loss occurs: from $\mathrm{B}$ to $\mathrm{C}$, for the first mass loss episode, and from shortly after $\mathrm{K}$ to $\mathrm{L}$, for most of the second Roche-lobe overflow. Note, however, that the final part of the second Roche-lobe overflow occurs when carbon has already been ignited in the core (see Sects. 3.4 and 4) and, thus, it is not shown in Fig. 2. As expected, the effects of the mass loss episodes considerably modify the evolutionary track when compared with the evolution of an isolated star of the same initial mass (García-Berro \& Iben 1994). For instance, although the descent along the red giant branch takes place at a slightly higher temperature than in the case of an isolated star (4400 K instead of $4300 \mathrm{~K}$ ), it is followed by a sudden shift to bluer regions of the diagram due to the adiabatic expansion and cooling that accompanies the first mass loss episode (see below). The second major different feature is that the developement of the blue loop during the core helium burning phase takes place at lower luminosities than in 
Table 1. Characteristics of models at various points in the H-R diagram of Fig. 2.

\begin{tabular}{cccccccccccc}
\hline Model & $t\left(10^{14} \mathrm{~s}\right)$ & $\log L$ & $\log T_{\text {eff }}$ & $\log R$ & $\log \rho_{\mathrm{c}}$ & $\log T_{\mathrm{c}}$ & $M_{\mathrm{He}}$ & $M_{\mathrm{C}}$ & $M_{1}$ & $M_{2}$ & $P_{\text {orb }}(\mathrm{yr})$ \\
\hline $\mathrm{A}$ & 6.1785 & 3.79 & 3.67 & 2.07 & 6.67 & 8.16 & 1.97 & 0.00 & 10.0 & $1.0-5.0$ & $0.1-1.0$ \\
$\mathrm{~B}$ & 6.1795 & 4.12 & 3.63 & 2.32 & 6.62 & 8.16 & 1.97 & 0.00 & & & \\
$\mathrm{C}$ & 6.1893 & 4.16 & 3.64 & 2.32 & 6.51 & 8.16 & 1.97 & 0.00 & 2.4 & $1.7-7.0$ & $0.3-0.5$ \\
$\mathrm{D}$ & 6.2917 & 3.99 & 3.64 & 2.23 & 6.45 & 8.18 & 2.04 & 0.00 & & & \\
$\mathrm{E}$ & 6.2936 & 3.96 & 3.59 & 2.32 & 6.45 & 8.18 & 2.04 & 0.00 & & & \\
$\mathrm{~F}$ & 6.2954 & 3.98 & 3.59 & 2.32 & 6.44 & 8.18 & 2.04 & 0.00 & & & \\
$\mathrm{G}$ & 6.3555 & 3.84 & 3.62 & 2.41 & 6.43 & 8.19 & 2.05 & 0.00 & & & \\
$\mathrm{H}$ & 6.8505 & 3.68 & 4.18 & 2.01 & 6.47 & 8.24 & 2.05 & 0.76 & & & \\
$\mathrm{I}$ & 7.2076 & 3.90 & 3.72 & 2.02 & 7.42 & 8.15 & 2.05 & 0.86 & & & \\
$\mathrm{~J}$ & 7.2122 & 3.82 & 3.81 & 1.85 & 7.43 & 8.42 & 2.05 & 0.91 & & & \\
K & 7.2178 & 3.89 & 3.64 & 2.18 & 6.43 & 8.45 & 2.06 & 0.93 & & & \\
$\mathrm{~L}$ & 7.2638 & 4.21 & 3.73 & 2.18 & 7.66 & 8.60 & 2.06 & 1.04 & 1.9 & $2.2-9.3$ & $0.1-1.0$ \\
\hline
\end{tabular}

Table 2. Surface abundances after the first dredge-up. SS stands for single star.

\begin{tabular}{lcccccc}
\hline Model & $X_{\mathrm{H}}$ & $X_{3}$ & $X_{\mathrm{He}}$ & $X_{\mathrm{C}}$ & $X_{\mathrm{N}}$ & $X_{\mathrm{O}}$ \\
\hline $10 M_{\odot}(\mathrm{SS})$ & 0.681 & $1.23 \times 10^{-5}$ & 0.305 & $1.79 \times 10^{-3}$ & $2.83 \times 10^{-3}$ & $7.71 \times 10^{-3}$ \\
$10 M_{\odot}(\mathrm{CBS})$ & 0.664 & $1.13 \times 10^{-5}$ & 0.324 & $1.64 \times 10^{-3}$ & $3.46 \times 10^{-4}$ & $7.19 \times 10^{-3}$ \\
$9 M_{\odot}(\mathrm{SS})$ & 0.696 & $1.49 \times 10^{-5}$ & 0.291 & $1.85 \times 10^{-3}$ & $2.48 \times 10^{-3}$ & $8.03 \times 10^{-3}$ \\
\hline
\end{tabular}

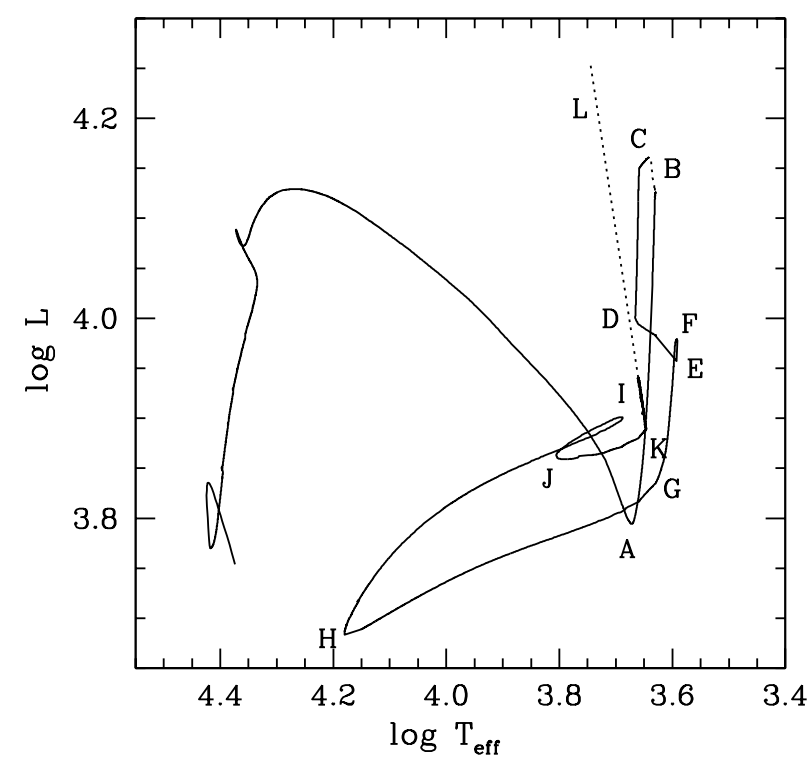

Fig. 2. Evolutionary track of the primary component in the Hertzsprung-Russell diagram. The physical quantities of the labeled models are shown in Table 1.

the case of the isolated model star $\left(6.9 \times 10^{3} L_{\odot}\right.$ instead of $\left.7.9 \times 10^{3} L_{\odot}\right)$.

In Fig. 3 we show the temporal evolution of the radius of the star (top panel) and of the convective regions associated with nuclear burning (bottom panel), from the zero age main sequence up to the off-center ignition of carbon in the helium-exhausted core. The inserts show with higher resolution the evolution of the convective regions and of the radius during the two mass-loss episodes. The initial central convective zone of Fig. 3 is due to the

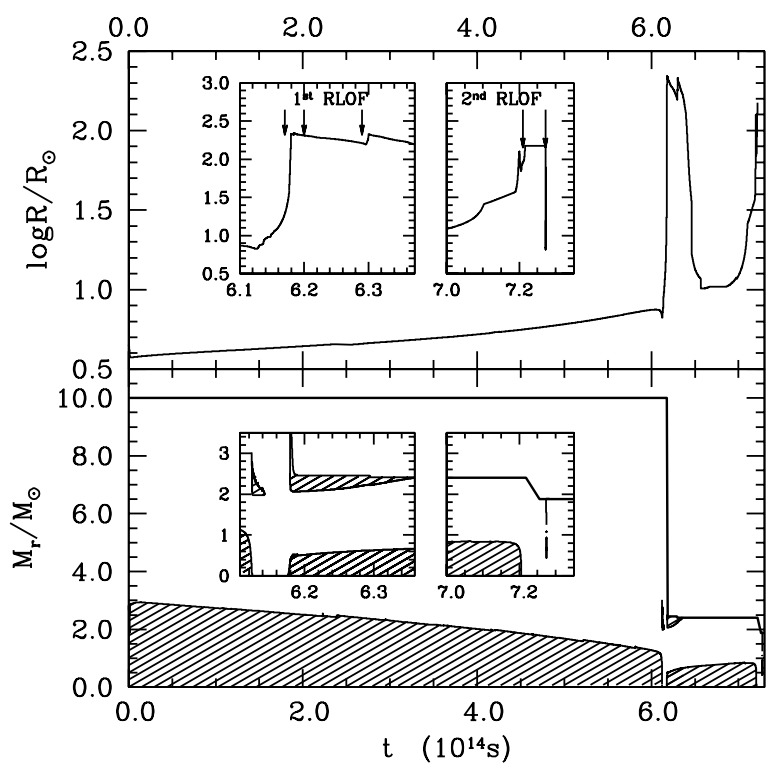

Fig. 3. Evolution of the main structure parameters as a function of time during the first and the second mass-loss episodes. The upper panel shows the evolution of the radius of the primary. The inserts show the mass-loss episodes with higher resolution. The lower panel shows the convective zones engendered during the evolution up to off-center ignition of carbon.

high fluxes engendered by the $\mathrm{CN}-$ cycle reactions and persists until hydrogen vanishes at the center. An off-center convective region forms later, very much in the same way as in the case of a single star. This convective region is due to the release of gravitational potential energy during the overall contraction phase that follows the exhaustion of central hydrogen and the establishement of the 
hydrogen-burning shell that occurs from points $\mathrm{C}$ to $\mathrm{D}$ in Fig. 2. The thick solid line in the lower panel corresponds to the total mass of the primary which, as it can be seen, decreases dramatically at the begining of the first dredge-up-process, which occurs simultaneously with the first Roche-lobe overflow. Since this mass loss episode occurs in the presence of a deep convective envelope, the associated time scales are short. On the contrary, we will see that the second mass-loss episode is much more stable since it is not associated with a dredge-up episode. This behaviour makes the two mass-loss episodes completely different and has important consequences. For instance, we expect to find a different pattern of surface composition after the first mass-loss episode and dredgeup, when compared to the evolution of a single star. The surface composition of both model stars and that of the isolated $9 M_{\odot}$ star just after the end of the dredge-up episode can be found in Table 2. As we shall show below, the comparison with the $9 M_{\odot}$ star is relevant for this study since some of the results obtained in the calculations reported here are much closer to the isolated $9 M_{\odot}$ model than to those of the $10 M_{\odot}$ single star. As can be seen, the helium and nitrogen contents are significantly higher in the case of a model star belonging to a close binary system.

\subsection{The first mass loss episode}

Our model undergoes a Case B mass loss process that starts shortly after hydrogen combustion in a shell has been established, and the surface radius reaches the Roche Lobe radius which, as previously mentioned, we have adopted to be $210 R_{\odot}$. In computing this mass-loss episode we have (somewhat arbitrarily) assumed that the primary keeps a constant radius which is coincidential with the Roche-lobe radius and that all the overflowing matter will be lost by the primary. Although this is a classical prescription there are, of course, other alternatives (Nelson \& Eggleton 2000). It is nevertheless worth noticing that when using the last approximation with secondary masses of around $\sim 5 M_{\odot}$, the Roche lobe radius changes by $\sim 10 \%$ and, consequently, we do not expect this to have a large impact on our results. We thus defer such study to a forthcoming publication.

In the first insert in the top and bottom panels of Fig. 3 the first mass-loss episode is shown. Also, in Fig. 4 we show the evolution of several interesting quantities like the hydrogen and helium nuclear luminosities. This mass-loss episode occurs in two distinct phases. The first and most violent phase occurs as the convective envelope is still advancing to the interior of the star and when helium has already been ignited at the center, leading to the formation of a central convective region, which reaches its equilibrium value when $\log \left(L_{\mathrm{He}} / L_{\odot}\right) \simeq 3.3$. This phase is clearly marked by the two arrows in the top insert of Fig. 3 and by the thin vertical lines of Fig. 4. During this first phase, the bulk of the hydrogen-rich envelope is lost $\left(\sim 7.6 M_{\odot}\right)$,

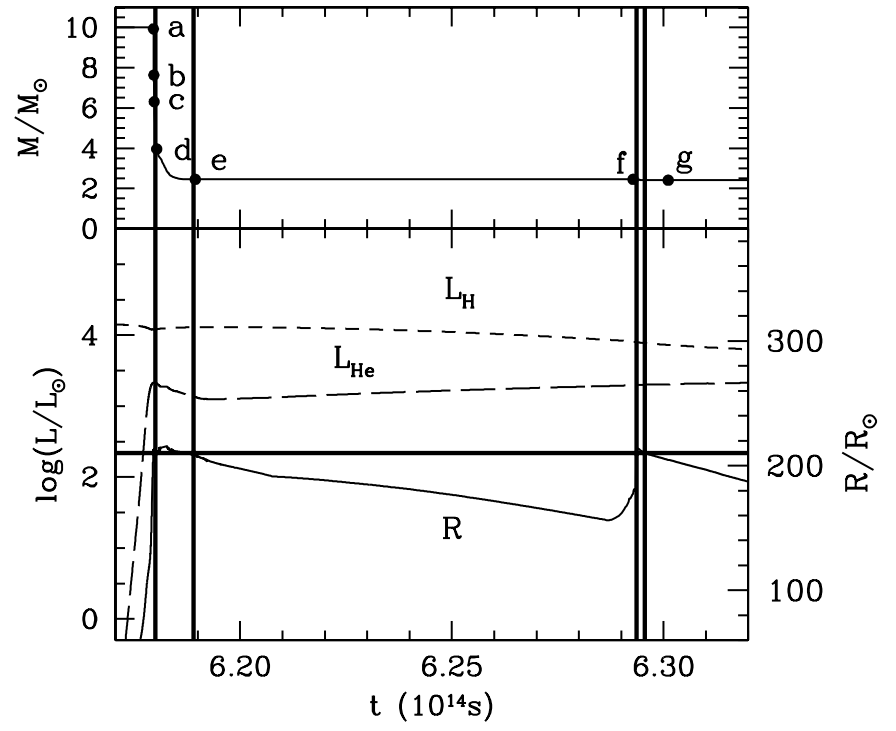

Fig. 4. Global characteristics of the primary component as a function of time during the first mass-loss episode. The upper panel shows the total mass as a function of time. The lower panel shows the surface radius and the luminosities provided by hydrogen and helium burning. The vertical thin lines delimit the two phases of mass loss. The surface luminosity is mostly provided by hydrogen combustion.

leaving a remnant of $2.4 M_{\odot}$ of which $\sim 0.5 M_{\odot}$ corresponds to the remaining $\mathrm{H}-$ rich convective envelope. The duration of this phase is $3.0 \times 10^{4} \mathrm{yr}$.

Since the dependence of the variation of the total radius of the star on the mass is $\Delta R \sim R M^{-1 / 3}$ (de Loore $\&$ Doom 1992), the mass-loss process experiences a positive feedback. Therefore, during the very first part of this mass-loss episode the feedback of the process allows very high values for the mass loss rates, that can reach values as high as $\dot{M}_{1} \sim 10^{-3} M_{\odot} \mathrm{yr}^{-1}$. At these very high mass-loss rates the star is no longer able to keep a constant radius and at the end of this phase the radius of the primary falls below the value of the Roche-lobe, and thus the mass transfer onto the secondary temporarily stops. As the evolution continues, the primary again fills its Roche-lobe radius, leading to a subsequent phase of mass-loss. During this second phase only $\sim 0.05 M_{\odot}$ are lost by the primary. Thus, a small portion of the $\mathrm{H}-$ rich envelope remains even at the end of the first mass-loss episode. The time scale for this second phase is significantly longer $\left(1.6 \times 10^{5} \mathrm{yr}\right)$, leading to much more modest mass-loss rates: $\dot{M}_{1} \sim 10^{-5} M_{\odot} \mathrm{yr}^{-1}$.

The existence of a deep convective envelope surrounding the $\mathrm{H}$-exhausted core of the primary and, consequently, the high values of the resulting mass-loss rates very much enhance the possibility that the system is embedded in a common envelope. No definite and accurate study of this kind of structure has been performed up to now. Thus, the part of the process in which a common envelope forms is plagued with many uncertainties, the most important one perhaps being the influence of the common envelope on the orbital parameters. 
The expressions provided by Eggleton (1983) and Paczyński (1971) can only be taken as approximations, or even upper limits, and it is necessary to rely on estimates that have been obtained for other systems that presumably have undergone a common envelope phase, like the cataclysmic variable $\mathrm{U}$ Gem. These estimates yield values for the angular momentum losses that can be as high as 70-90\% (Iben \& Tutukov 1984). In any case, when a common envelope is present, the angular momentum losses are expected to be high due to the release as sound waves of the energy generated by frictional drag in the matter of the envelope. Therefore, from an initial separation between the components of $A_{\mathrm{i}} \sim 350-450 R_{\odot}$ (corresponding to $1 M_{\odot} \leq M_{2} \leq 5 M_{\odot}$ respectively) one can assume a typical $80 \%$ angular momentum loss and apply the equation

$J_{\text {orb }}^{2}=G A_{\mathrm{i}} \frac{\left(M_{1} M_{2}\right)^{2}}{M_{1}+M_{2}}$

to get the final separation between components after the first Roche-lobe overflow, $A_{\mathrm{f}} \sim 150-350 R_{\odot}$. It should be noted, nevertheless, that this way of estimating the final orbital parameters usually leads to larger orbital separations than the values obtained when using the treatment of Webbink (1984).

We can estimate the ratio between the mass accreted by the secondary and the mass lost by the primary, $\beta$, by assuming stable accretion onto the secondary. Thus, $\dot{M}_{2}=$ $M_{2} / \tau_{\mathrm{KH}}$, where $\tau_{\mathrm{KH}}$ is the Kelvin-Helmholtz timescale. Considering typical values for the luminosity and for the surface radius of a main sequence companion of mass between 1 and $5 M_{\odot}$, we get a reasonable value for the mass accretion rate of the secondary, $\dot{M}_{2} \sim 10^{-4} M_{\odot}$. Taking into account that most of the mass is lost by the primary during the first $3.0 \times 10^{4} \mathrm{yr}$, we can easily calculate an average value for $\beta$ during this mass loss episode, which turns out to be $\beta \sim 0.1$. Of course, it can be argued as well that another reasonable way to estimate $\beta$ is to adopt the Eddington limit instead of the thermal timescale, this being a firm upper limit. For the range of relevant parameters, this procedure would lead in our case to accretion rates 3 times larger, or equivalently, to $\beta \sim 0.3$. Nevertheless it should be noted that the calculations of Hjellming \& Taam (1991) predict a significantly lower value of $\beta$, of the order of 0.01 . Thus, the mass accreted by the secondary will be in the range $0.7-2.0 M_{\odot}$, at most. Since the main sequence lifetime of a $7.0 M_{\odot}$ star is $t \sim 9.0 \times 10^{14} \mathrm{~s}$ it is clear that reversal mass transfer will not be enabled until carbon is exhausted in the inner core of the primary (see Table 1 and Sect. 4 below), in accordance with our scenario.

We have considered the possibility that part of the matter ejected by the primary component might be accreted back by the same star. In order to obtain some hints of the possible outcomes to this problem, we have estimated the duration of the common envelope phase as in Iben \& Tutukov (1984) and compared it with the thermal time scale of the primary. As the latter is much longer

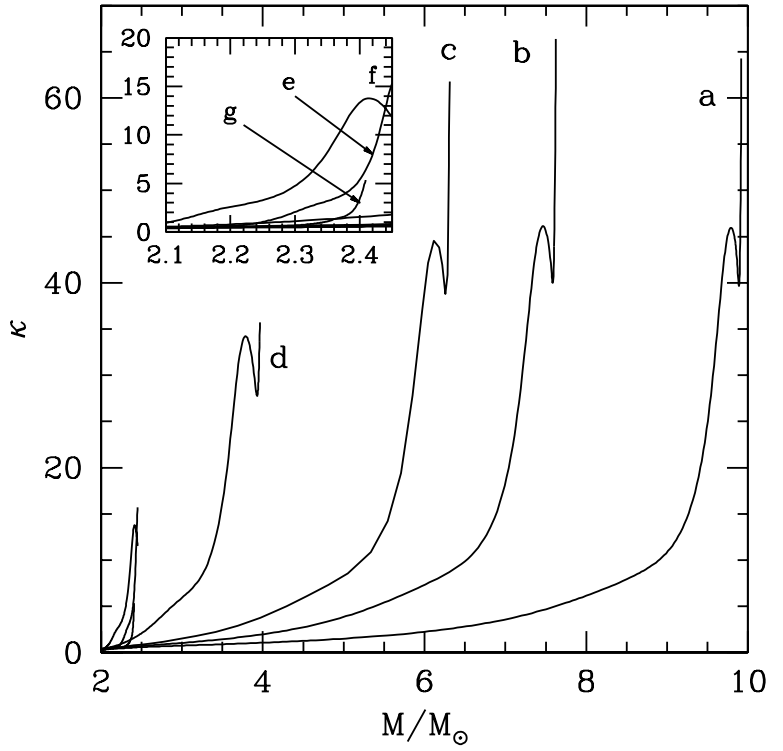

Fig. 5. Opacity profiles in the hydrogen-rich envelope during the first Roche lobe overflow. The models displayed correspond to the different times labeled in Fig. 4. The insert shows, with higher resolution, the opacity profiles for the last models.

than the expected time for the common envelope to remain bound to the system, there are strong reasons to admit that there is not enough time for the ejected gas to cool down, lose kinetic energy and be overtaken by the gravitational potential of the primary.

It is also worth noting that the duration of the first dredge-up episode is longer in the calculations reported here than in the case of the isolated model described in García-Berro \& Iben (1994). This is due to the fact that the fast release of gravitational energy during the compression phase that happens in the middle of the first mass loss episode cannot be evacuated solely by radiation and, thus, allows convection to persist. On the other hand, one should not forget that the overall evolutionary time scales are longer for decreasing masses and this effect would also have an influence on the duration of the dredge-up episode.

In order to find an explanation that, at least partially, accounts for the behaviour of the envelope during the first mass loss episode, in Fig. 5 we show the opacity profiles of several specific models for the times labeled in Fig. 4 (models a to g). The fastest expansion phase is coincidential with models a to $\mathrm{c}$, in which the existence of a deep convective envelope, basically composed of hydrogen at relatively low temperature, leads to a high opacity and, thus, to an inefficient transport of the energy. Therefore, an important portion of the energy generated at the hydrogen burning shell is not driven outwards but, instead, it is transformed into internal energy in the envelope and, then, into work of expansion, thus keeping the value of the surface radius very close to the Roche lobe radius.

When the model reaches approximately $4 M_{\odot}$ (model d), a large portion of the hydrogen-rich envelope is already lost, and the inner and hotter layers are exposed. 
At this point, on the one hand we have less mass able to absorb the flow of energy and, on the other, the opacity is also smaller. Consequently, both phenomena allow nuclear energy to flow almost freely to the surface without being transformed into work of expansion and, at model e, when the mass of the star is about $2.45 M_{\odot}$, a fast overall (almost adiabatic) contraction of the convective envelope occurs, leading the surface radius to drop below the value of the Roche lobe and, hence, mass loss temporarily halts. Finally, for model $f$ the opacity increases due to compression and, thus, a new phase of expansion occurs which drives the surface radius to again reach the value of the Roche lobe radius. Thus the mass loss episode is restored for a brief interval, until some more cool hydrogenrich layers are lost. Finally the opacity definitely decreases (model g) and the mass loss episode is finished.

It is worth mentioning at this point that we get a remnant of slightly higher mass than that of the models found in the existing literature (Iben \& Tutukov 1985; Domínguez et al. 1994). This is due to the fact that in our model the hydrogen-rich envelope is not completely lost during the first Roche lobe overflow episode. However, since the rest of the remaining hydrogen-rich envelope is lost later during the second Roche lobe overflow episode, and since the growth of the helium core is very small during the time between the two mass loss episodes (approximately $0.08 M_{\odot}$ ), we expect that the influence on the $\mathrm{CO}$ and $\mathrm{ONe}$ core sizes and compositions is negligible and so the final results will not be substantially different.

\subsection{The second mass loss episode}

The second mass loss episode starts when helium burning begins in a shell and, as a consequence, its surface radius approaches again that of the Roche lobe (see Fig. 3), which now is $\sim 80 R_{\odot}$. The global characteristics (the mass and radius and the luminosities associated with the active burning regions) of the primary during the second mass loss episode are shown in Fig. 6. The fact that in the outer envelope, from which the matter is removed, energy is transported by radiation instead of being transported by convection, allows the process to take place in a more stable way than in the first mass loss episode. Hence, the mass loss rates are much smaller $\left(\dot{M}_{1} \sim 10^{-6} M_{\odot} \mathrm{yr}^{-1}\right)$ during most of the process, except at two phases, during which the values of the mass loss rate are one order of magnitude higher. The first phase corresponds to the beginning of the mass loss episode, when the remainder of the hydrogen-rich envelope is lost, and the second phase happens nearly at the end of the process, when carbon is ignited off-center. The end of the process is determined by a steep decrease in the surface radius of the star that takes values below the Roche lobe radius.

The moderate values we get for the mass loss of the primary support the hypothesis of conservative mass transfer. Furthermore, one can compare the luminosity associated with accretion onto the secondary, which is given by
$L_{\text {acc }}=\dot{M}_{2}\left(\Phi_{L_{1}}-\Phi_{R_{2}}\right)$, where $\Phi_{L_{1}}$ and $\Phi_{R_{2}}$ stand for the gravitational potential computed at the Roche lobe radius and at the radius of the secondary respectively, with the Eddington luminosity of the secondary, $L_{\text {Edd }}$. For a set of typical values for the secondary star, we get that $L_{\text {acc }} \ll L_{\text {Edd }}$ and, thus, according to Han et al. (1999) conservative mass transfer is most likely.

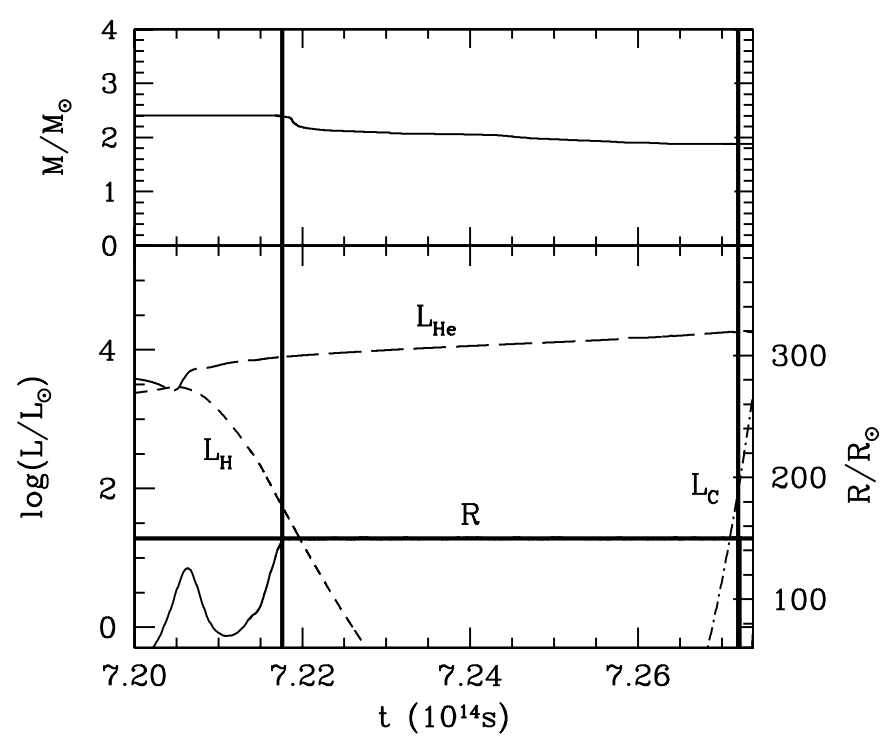

Fig. 6. Global characteristics of the primary component as a function of time during the second mass loss episode. The upper panel shows the total mass as a function of time. The lower panel shows the surface radius and the luminosities provided by hydrogen, helium and carbon burning. The vertical thin lines delimit the second mass loss episode. The surface luminosity is mostly provided by helium combustion.

\subsection{Overall characteristics before carbon ignition}

In Fig. 7 we show the evolution of the center of the primary in the $\log \rho-\log T$ diagram from the main sequence until the onset of carbon ignition (solid line), and the evolution of the center of the isolated $10 M_{\odot}$ star (dashed line). As one can expect from the previous discussion, the differences between both cases start to show up at the onset of helium burning at the center, which, as explained above, is almost coincident with the first mass loss episode. The evolution of the primary component of the close binary system leads to higher central densities for the same value of the temperature than its isolated counterpart. In fact, mass loss has two obvious consequences. Firstly, the size of the central helium-burning convective region is smaller for the case studied here than in the evolution of isolated $10 M_{\odot}$ star. Secondly, the mass of the primary component of the binary system is much smaller than its isolated counterpart. Accordingly, the He-exhausted core of the primary is smaller $\left(\sim 1.05 M_{\odot}\right)$ and with higher central densities than the core resulting from the evolution of a single $10 M_{\odot}$ star $\left(\sim 1.15 M_{\odot}\right)$ and very similar to that of a $9 M_{\odot}$ isolated $\operatorname{star}\left(\sim 1.04 M_{\odot}\right)$. 


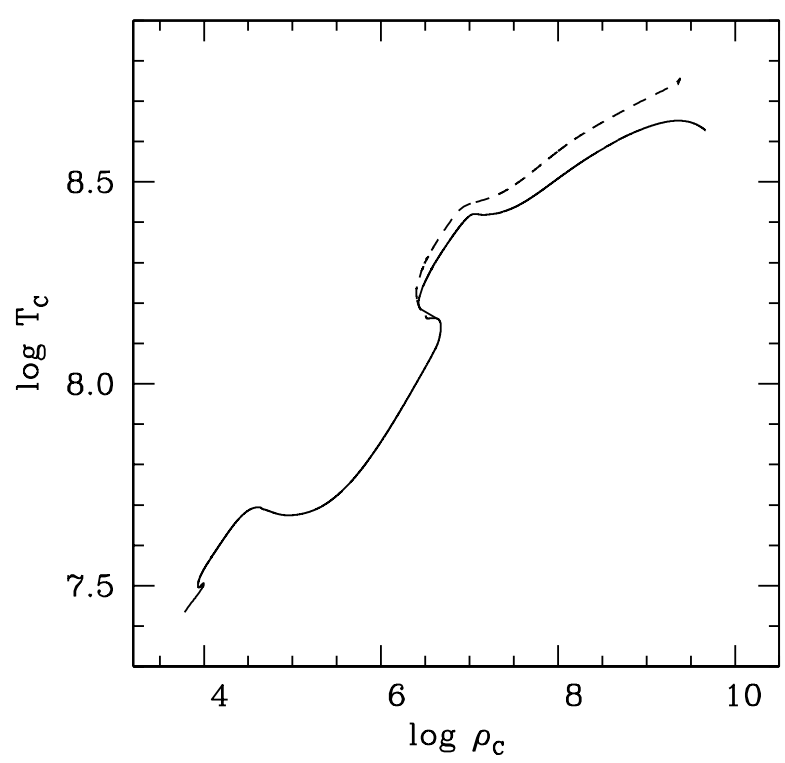

Fig. 7. Evolution in the $\log \rho-\log T$ plane of the central layer of the isolated $10 M_{\odot}$ star (dashed line) and the of the primary component of the close binary system studied here (solid line), until the onset of carbon burning.

The abundances in the central regions of the core are also affected by mass loss in a similar way, and so, the resulting composition is more similar to that of the single $9 M_{\odot}$ model. At the end of the second mass loss episode the mass of the primary is $1.88 M_{\odot}$, of which the innermost $1.05 M_{\odot}$ corresponds to a carbon-oxygen core which is surrounded by a helium-rich envelope. The chemical composition profiles at this moment are shown in Fig. 8. As can be seen in this figure the abundance profiles of our model before carbon ignition reveal a higher carbon and neon content, and lower amounts of oxygen and magnesium than those of the single $10 M_{\odot}$ model. Also the helium burning shell is narrower in the case studied here, due to the fact that the (helium) envelope over this burning shell is much smaller.

\section{The carbon burning phase}

The carbon burning phase takes place under conditions of partial degeneracy and - as one can expect from the comparison between the characteristics of the primary star in a close binary system and that of single star models of similar mass before carbon burning - many similarities are also found when analysing this phase of the evolution. Actually, the most prominent features, such as the carbon flashes and the convective regions associated with each one of these (see Fig. 9) are very similar for the case studied here and those of a $9 M_{\odot}$ model star. These flashes reach about $10^{7}-10^{8} L_{\odot}$, and last for approximately $10^{3} \mathrm{yr}$. However, as it happens in the case of the evolution of isolated stars of this mass range, during most of the carbon burning phase the surface luminosity remains almost constant and close to a value of $\log \left(L / L_{\odot}\right)=4.3$.

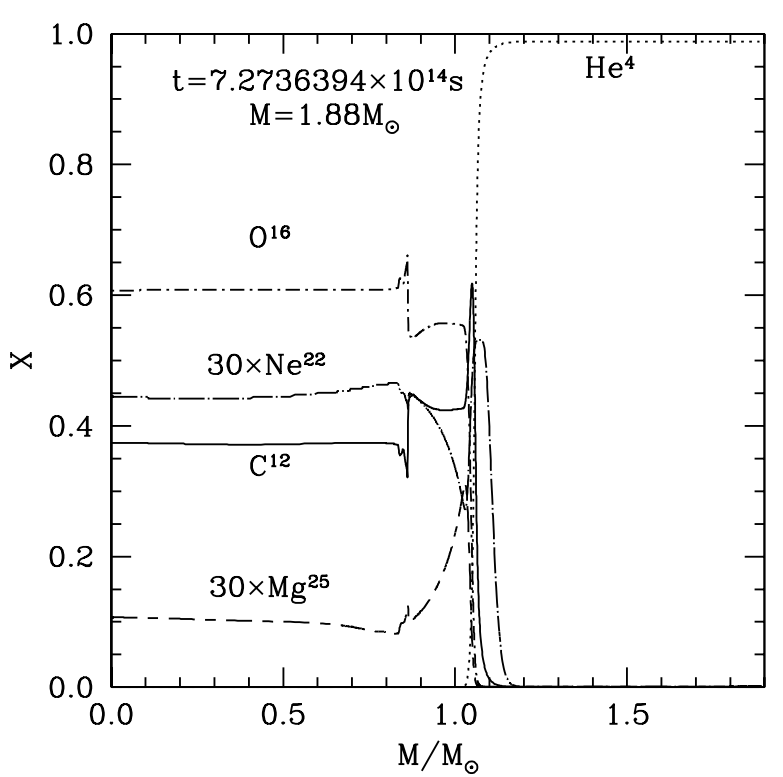

Fig. 8. Abundance profile of the core of the primary at the end of the second mass loss episode, which is practically coincidential with the beginning of carbon burning.

The first flash is a prototypic one. As in the case of isolated stars within this mass range, carbon is ignited offcenter due to neutrino cooling of the central regions. The mass coordinate at which carbon is ignited is $\sim 0.42 M_{\odot}$, very close to the value obtained for the single $9 M_{\odot}$ star $\left(\sim 0.43 M_{\odot}\right)$. As carbon luminosity increases, most of the energy generated by nuclear reactions is used to increase the temperature of the adjacent layers, thus forcing high temperature gradients, which ultimately lead to the formation of a convective zone that allows a more efficient transport of energy. This increase in the temperature is followed by an expansion of the nearby layers and in particular, of the helium burning shell, which cools down and, hence, its luminosity temporarily decreases. Apart from the formation of the inner convective shell, the flash also affects the location of the inner edge of the convective envelope that moves deeper into the star when the carbon luminosity increases and receeds when the carbon luminosity decreases again, very much in the same fashion as in the isolated $9 M_{\odot}$ star.

The second flash occurs at a smaller mass coordinate (carbon is re-ignited at the point where the penetration of the inner edge of the previous convective shell was maximum) and the physical mechanisms operating are very similar to those of the first one. However, its maximum strength is considerably smaller $\left(\sim 10^{7} L_{\odot}\right.$ instead of $\left.\sim 10^{8} L_{\odot}\right)$. After the most violent phase of this flash is over, the carbon luminosity does not decrease below that of helium but, rather, keeps a stationary value, $\log \left(L_{\mathrm{C}} / L_{\odot}\right) \simeq 4.6$, which is slightly larger than the value of the helium luminosity, $\log \left(L_{\mathrm{He}} / L_{\odot}\right) \simeq 4$. During this phase the carbon-burning flame propagates inwards at a roughly constant speed - see García-Berro et al. (1997) for a detailed description of the energy balance 


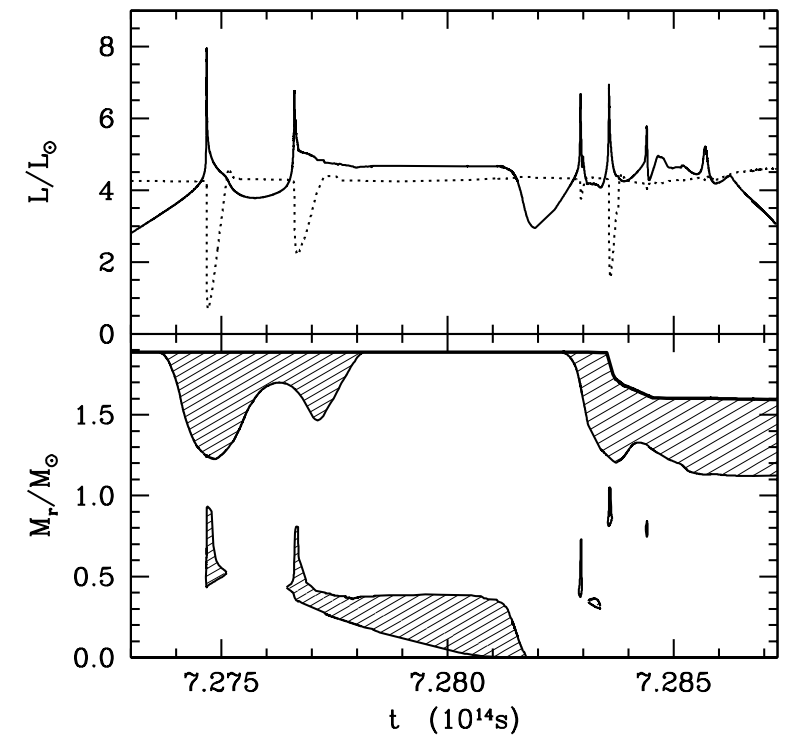

Fig. 9. Evolution of the nuclear luminosities during the carbon-burning phase (upper panel), and of the associated convective regions (lower panel). The solid and the dotted lines in the upper panel correspond, respectively, to the carbon and helium luminosities.

established during this phase - reaching the center at $t \simeq$ $7.282 \times 10^{14} \mathrm{~s}$.

A very distinctive feature of the case studied here when compared to the evolution of an isolated $9 M_{\odot}$ star is that at $t \simeq 7.278 \times 10^{14} \mathrm{~s}$ the outer convective envelope disappears as the carbon burning front advances to the center. In fact, the second dredge-up in the isolated $9 M_{\odot}$ star is caused by the expansion and cooling of the layers just below the base of the convective envelope (García-Berro et al. 1997), which allows the increase of the radiative temperature gradient in this region and, so, the inner advance of convection down to $M_{\mathrm{r}} \simeq 1.2 M_{\odot}$. After that, the base of the convective envelope remains at an approximately constant position, as the energy supplied by the helium burning shell and the opacity of the nearby layers can keep the temperature gradient high enough. In the case studied here, the base of the convective envelope reaches the same position as in the single star due to a similar mechanism, but, unlike the case of the isolated $9 M_{\odot}$ star, this position cannot be maintained, instead, it receeds and disappears. The reason for this behaviour can be explained with the help of Figs. 10 and 11, where we show some relevant structural and dynamical quantities for times $t=7.277763 \times 10^{14} \mathrm{~s}$, just before the disappearance of the convective envelope, and $t=7.278391 \times 10^{14} \mathrm{~s}$, just after the convective episode.

Panel c of Fig. 10 shows that there are three interesting and distinct regions in the star. The first of these regions is below the helium discontinuity and there, the work of expansion fed by carbon burning is devoted basically to lift degeneracy and, thus, we have expansion at almost constant temperature (panel b). On top of this region we have the helium burning shell where the energy supplied by nu-

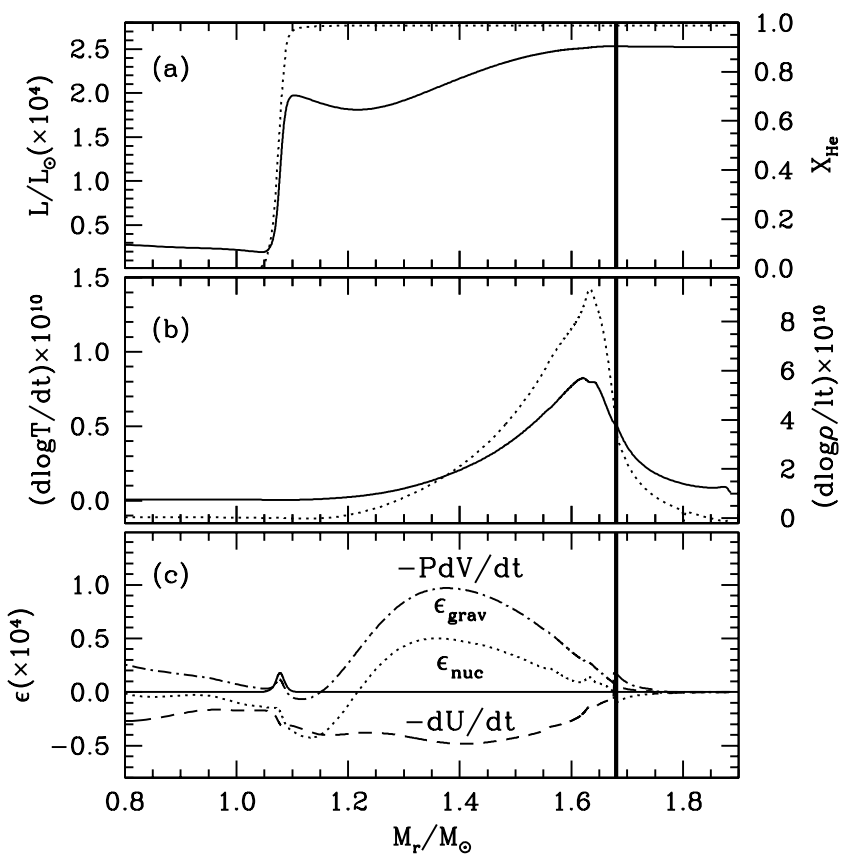

Fig. 10. Relevant structural and dynamical quantities for a model at time $t=7.277763 \times 10^{14} \mathrm{~s}$, just before the disappearance of the convective envelope, in a region extending from below the helium-burning shell until the surface of the star. Panel a) velocity (solid line) and helium profile (dotted line). Panel b) time variation of the temperature (solid line) and density (dotted line). Panel c) energy release rates (nuclear and gravothermal) along with the time derivative of the internal energy and the work of expansion. The nuclear energy release rate has been divided by $10^{2}$ in order to fit into the scale. The thin solid line marks the position of inner edge of the convective envelope.

clear reactions is used to build up the luminosity profile (panel a). In the region between the helium burning shell and the base of the convective envelope, the flux is partially transformed into heating and, at the same time, the whole region is collapsing. This in turn causes a large temperature gradient at $M_{\mathrm{r}} \simeq 1.7 M_{\odot}$. As the evolution continues, the temperature in this region steadily increases. Thus, the temperature gradient ultimately flattens and the inner edge of the convective envelope consequently receeds. Panel c of Fig. 11 shows that the nuclear energy released by the helium burning shell remains the same, but now most of this energy merely flows outwards (panel a), rather than being transformed into work of expansion. Instead, the whole region on top of the helium burning shell is compressed leading to a non-homogeneous increase of the temperature (panel b) which effectively erases the temperature gradient and forces the disappearance of the convective region. This translates into an increase in the luminosity for $M_{\mathrm{r}} \gtrsim 1.4 M_{\odot}$.

When carbon-burning in the central regions is over, a series of small shell burning episodes occurs (Fig. 9). Each one of these episodes has a decreasing strength and leads to the almost complete exhaustion of carbon in a core of $1.05 M_{\odot}$. It is interesting to note that, unlike what 


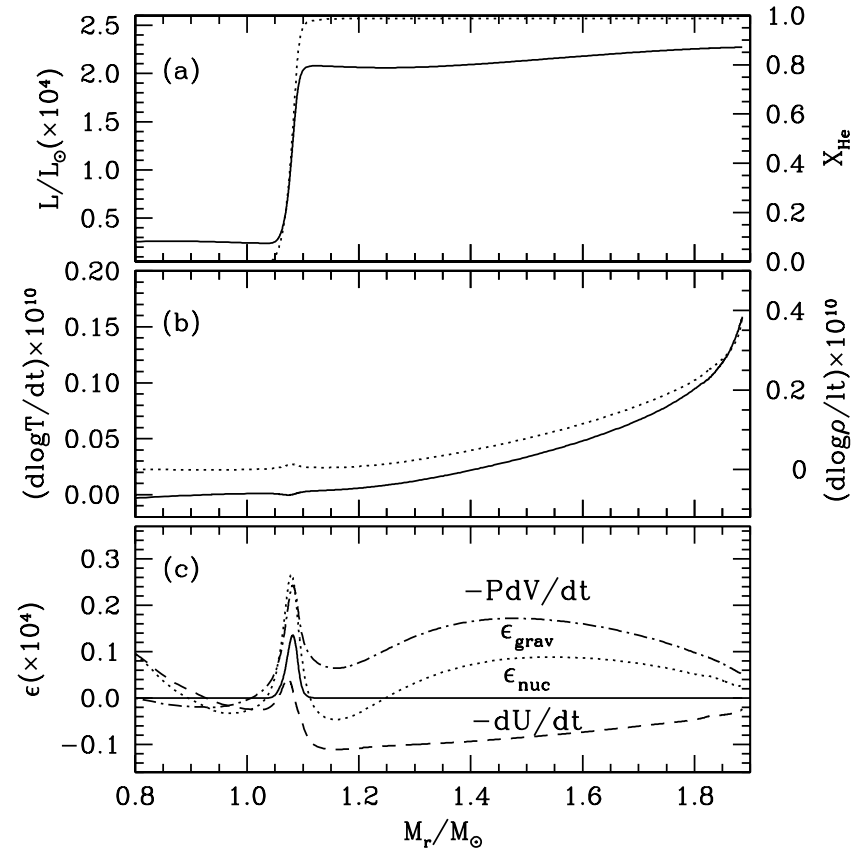

Fig. 11. Same as figure 10, but for time $t=7.278391 \times 10^{14} \mathrm{~s}$, just after the convective episode.

happens with the rest of these mild flashes, the first of these is not accompanied by a substantial decrease in the helium luminosity. The reason for this behaviour is twofold. Firstly, the duration of this flash is smaller and, hence, less energy is released. Secondly, and most important, there exists a thick radiative layer (of about $0.7 M_{\odot}$ ) between the initial location of the convective carbonburning shell and the helium-burning shell. Therefore, the energy is almost totally absorbed before reaching the helium-burning shell and, thus, no expansion nor cooling of the helium burning shell are produced. During all this phase the core contracts, and the outer layers of the star begin a new expansion as the outer convective envelope reappears and its inner edge advances to the interior of the star. However, the radius of the star remains below the Roche lobe radius and only exceeds its value at the end of the second of these flashes.

Since, as discussed above, the second mass loss episode was most probably conservative and during the bulk of the carbon burning phase in the central regions (say $t \lesssim 7.282 \times 10^{14} \mathrm{~s}$ ) the model star keeps its radius below that of the Roche lobe, the orbital parameters remain unaffected. As explained before, only at the very end of the carbon burning phase the flash activity is accompanied by a rapid increase of the surface radius of the star beyond the Roche lobe radius. This, of course, translates into a new mass loss episode for $t \gtrsim 7.283 \times 10^{14} \mathrm{~s}$. This mass loss episode lasts for a short period of time, of about $\sim 2 \times 10^{11} \mathrm{~s}$ and the mass loss rates are of $\sim 10^{-4} M_{\odot} \mathrm{yr}^{-1}$ at the beginning of the episode, becoming much smaller as the evolution proceeds. After this short phase of Roche lobe overflow, the radius of the primary star turns out to be very large, but its rate of increase slows down considerably. At

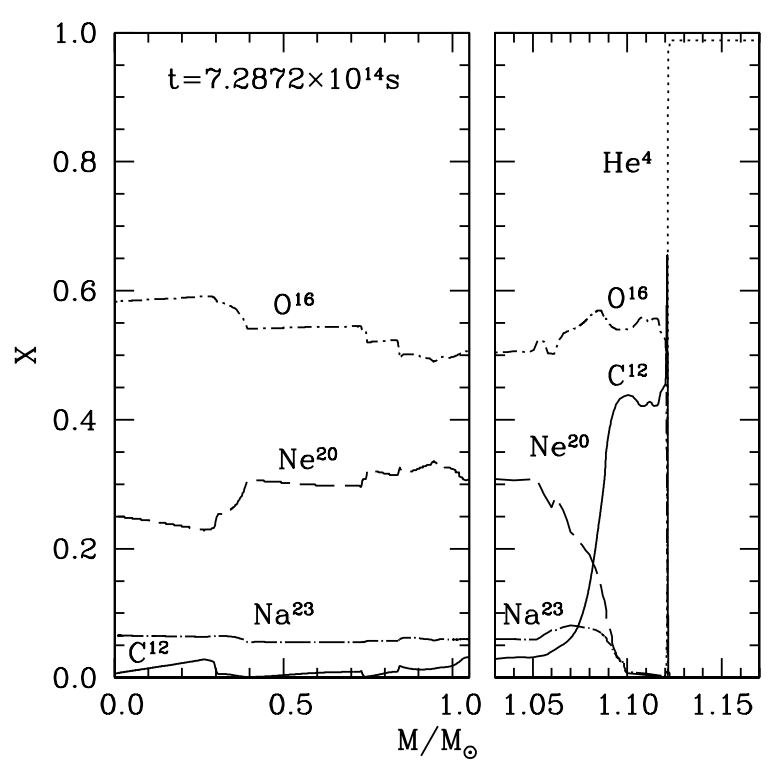

Fig. 12. Final abundances of the primary star after the carbon burning phase. Note the existence of a carbon-rich buffer on top of the carbon exhausted core.

this evolutionary stage, the density of the extended envelope is so small that a stellar wind could also drive the loss of the remaining envelope. In any case, stellar winds play a significant role which can be comparable to Roche lobe overflow. Thus, we assume that a stellar wind removes mass from the surface following closely the parametrization of Nieuwenheuzen and de Jager (1990) which gives typical rates of $\sim 5 \times 10^{-7} M_{\odot} \mathrm{yr}^{-1}$ (this is the rate considered in Fig. 9). However, since these mass loss rates are poorly known, we have conducted a series of numerical experiments where we have changed the mass loss rate from $10^{-5} M_{\odot} \mathrm{yr}^{-1}$ to $10^{-7} M_{\odot} \mathrm{yr}^{-1}$ and we have found essentially the same results for the very final part of the carbon burning phase, except of course for the mass of the remaining helium envelope.

The exhaustion of central carbon is not complete but, instead, at the innermost $0.3 M_{\odot}$ there remains remains a small amount of unburnt carbon, reaching a maximum abundance of $X_{\mathrm{C}}=0.025$ at $M_{\mathrm{r}}=0.25 M_{\odot}$ (see Fig. 12). Analogous to what was found in the series of papers devoted to the evolution of isolated stars within this mass range, most of the ashes of carbon burning are sodium, neon and oxygen, the abundance of magnesium being very small. This could have important consequences for the accretion-induced collapse scenario (Gutiérrez et al. 1996). It is also noteworthy that the relatively thick carbon-rich buffer is located just below the helium envelope, which could make the resulting He-rich white dwarf practically indistinguishable from an observational point of view from a regular massive carbon-oxygen white dwarf (Weidemann 2000). 


\section{Summary and discussion}

We have followed the evolution of a $10 M_{\odot}$ primary component of solar metallicity belonging to a close binary system with a secondary of low or intermediate-low mass, from its main sequence phase until carbon is exhausted in the core and a degenerate remnant is formed. Our main goal consisted of extending the previously existing calculations of this kind of system in order to follow in full detail the carbon burning phase, which at present has not yet been well studied. We have found that the primary star undergoes three mass-loss episodes. The first of these episodes occurs after hydrogen exhaustion in the core and most problably is not conservative, due to the existence of a deep convective envelope which leads to the formation of a common envelope. The second happens after helium exhaustion, and it is most problably conservative. Finally, the third one occurs when carbon burning in a series of succesive shells sets in. We have analysed the changes in the structure and composition that the primary star suffers while simultaneously undergoing carbon burning and mass loss. The determination of the mass loss rates at this very late stage of the evolution, whether due to Roche lobe overflow, or to stellar winds, is still an open question, and therefore, we have explored a broad range of mass loss rates ranging from $10^{-7}$ to $10^{-5} M_{\odot} \mathrm{yr}^{-1}$ in order to understand how the uncertainties affect the gross properties of the resulting degenerate object. We have found that our incomplete knowledge of the mass-loss rate does not affect the final result as far as the core is concerned. In particular, carbon is not reignited. Thus, the final characteristics of the remnant are well determined. After the evolution through these different burning stages and under the influence of a close companion, the initially $10 M_{\odot}$ star will form a $\sim 1.1 M_{\odot}$ degenerate remnant, with an oxygenneon core of $\sim 0.9 M_{\odot}$ surrounded by a carbon-oxygen rich mantle of $\sim 0.2 M_{\odot}$ and a thin helium envelope.

We have found remarkable differences between our results and those found by other authors that have also followed the evolution of a $10 M_{\odot}$ model star up to the carbon burning phase. For instance, Nomoto $(1992,1984)$ obtained a final core of larger mass $\left(\sim 1.3 M_{\odot}\right)$ with significantly higher values for the abundances $\mathrm{Ne}^{20}$ and $\mathrm{Mg}^{24}$, and lower values for the $\mathrm{O}^{16}$ and $\mathrm{Na}^{23}$ abundances. The ultimate reason for this difference is that the nuclear reaction rates used by Nomoto (Fowler et al. 1975) are different from ours (Caughlan \& Fowler 1988). In this regard, it is important to recall here that in this paper we have chosen the same physical inputs as in Ritossa et al. (1996) in order to remain consistent with our previous calculations. Since then there have been some new determinations of the thermonuclear reaction rates, particularly the NACRE compilation (Angulo et al. 1999). However, for the most important reaction channels involved in carbon burning, the differences are not expected to be large and, thus, the chemical composition of the core can be considered as relatively safe, perhaps the most important expected difference being an even smaller amount of $\mathrm{Mg}^{24}$
(Palacios et al. 2000; José et al. 1999). The comparison with the ONe core obtained by Domínguez et al. (1993) it is not easy either, since they start from a different initial $\mathrm{CO}$ core prior to carbon burning. Moreover, they removed the outer envelope during the carbon burning phase and, hence, their treatment for this phase differs substantially from ours. This, in particular, could be the reason for the central region of unburnt carbon which is quite apparent in their results, although poor mass resolution of the central regions - see the discussion in García-Berro et al. (1997) cannot be totally discarded. Regarding the average abundances of the ONe core, they obtained, using similar nuclear reaction rates, $X\left(\mathrm{O}^{16}\right)=0.72, X\left(\mathrm{Ne}^{20}\right)=0.25$ and $X\left(\mathrm{Mg}^{24}\right)=0.03$. The high value of the $\mathrm{O}^{16}$ abundance is surprising, whereas the rest of the abundances are similar. Moreover, they did not find $\mathrm{Na}^{23}$, which is an important isotope in our calculations. Thus, all these differences could be due to the use of a simplified nuclear network, much smaller than ours. In summary, the composition of our ONe core differs from the former results, especially for the $\mathrm{Mg}^{24}, \mathrm{Na}^{23}$ and $\mathrm{Ne}^{20}$ nuclei, which are the elements onto which electron captures happen and, consequently, may substantially affect the determination of the explosive ONe ignition density. The fact that the mass of the ONe core found by these authors $\left(\sim 0.93 M_{\odot}\right)$ is quite similar to that obtained here is, however, encouraging.

The definite final result is not necessarily an oxygenneon white dwarf, because the evolution of the entire system has not stopped yet. In fact, as the secondary component evolves, it could reach such a dimension that its radius could exceed that of its Roche lobe, and mass transfer could take place onto the remnant. Depending on the evolution of the orbital parameters and, ultimately, on the mass transfer rates, different possibilities arise:

1. A cataclysmic variable could be formed, if the accretion rates are below the critical rates for hydrogen burning. At typical accretion rates of about $10^{-9} M_{\odot} \mathrm{yr}^{-1}$ onto such a white dwarf, the amount of hydrogen-rich material from the secondary that must be accreted before an outburst occurs ranges between $10^{-4}$ - see, for instance, José \& Hernanz (1998) and references therein - and $10^{-5} M_{\odot}$ (Schwartzman et al. 1994). If we accept the results of Iben \& Fujimoto (1992) that significant hydrogen abundance extends in a mixture with the degenerate material down to a distance of about $10^{-5} M_{\odot}$ under the surface, and that approximately $2 \times 10^{-5} M_{\odot}$ are expelled during each outburst, we conclude that between 3000 and 4000 outburts (in a total time of at least 3-4 Myr) must occur before the white dwarf is definitely deprived of its $0.035 M_{\odot} \mathrm{CO}$ rich layer, and so, before significant amounts of $\mathrm{Ne}^{20}$ can be detected in the expelled material.

2. If stable accretion is possible, the degenerate object can increase its mass up to the Chandrasekhar mass, finally leading to a weak supernova explosion, activating the accretion-induced collapse scenario and leaving a 
neutron star. A possible observational counterpart for this outcome could be the massive radio pulsar PSR J0045-7319 (Kaspi et al. 1994), which belongs to a binary system in which its companion is an intermediate mass $\left(M \gtrsim 4 M_{\odot}\right)$ B star. More evolved systems may include an intermediate mass binary system consisting of a pulsar and a white dwarf. In this sense, up to seven pulsars with these characteristics have recently been discovered (Edwards \& Bailes 2001). In particular, we consider PSR J1756-5322 as a very likely counterpart because the mass of the white dwarf component is $\gtrsim 0.55 M_{\odot}$.

3. Finally, if the mass transfer rates are so large that the white dwarf is not able to accrete all the mass lost of its companion, a common envelope would form again, and most of the mass would be lost by the system, leaving an ONe white dwarf belonging to a close binary system. A possible observational counterpart to this outcome is IK Peg (Smalley et al. 1996), which is a confirmed binary system composed of an unevolved star plus a massive white dwarf $\left(M_{\mathrm{WD}} \simeq 1.15 M_{\odot}\right)$, possibly made of oxygen and neon.

Acknowledgements. Part of this work was supported by the Spanish DGES project number PB98-1183-C03-02, by the MCYT grant AYA2000-1785, by the CIRIT and by Sun MicroSystems under the Academic Equipment Grant AEG-7824-990325-SP. We also wish to thank J. José for carefully reading the manuscript and to our referee (P. P. Eggleton) for his very valuable comments and suggestions.

\section{References}

Angulo, C., Arnould, M., Rayet, M., et al. 1999, Nucl. Phys. A, 656,3

Caughlan, G. R., \& Fowler, W. A. 1988, Atom. Data Nuc. Data Tables, 40, 283

De Loore, C., \& de Greve, J. P. 1992, A\&AS, 94, 453

De Loore, C., \& Doom, C. W. H. 1992, Structure and evolution of single and binary stars, Astrophysics and Space Science Library, vol. 179 (Dordrecht: Kluwer)

De Loore, C., \& Vanbeveren, D. 1992, A\&A, 260, 273

De Loore, C., \& Vanbeveren, D. 1994, A\&A, 292, 463

De Loore, C., \& Vanbeveren, D. 1995, A\&A, 304, 220

Domínguez, I., Tornambé, A., \& Isern, J. 1993, ApJ, 419, 268

Edwards, R. T., \& Bailes, M. 2001, ApJ, 547, L37
Eggleton, P. P. 1983, ApJ, 268, 368

Fowler, W. A., Caughlan, G. R., \& Zimmermann, B. A. 1975, ARA\&A, 13, 69

Fujimoto, M. Y., \& Iben, I. 1992, ApJ, 399, 646

García-Berro, E., \& Iben, I. 1994, ApJ, 434, 306

García-Berro, E., Ritossa, C., \& Iben, I. 1997, ApJ, 485, 765

Gutiérrez, J., García-Berro, E., Iben, I., et al. 1996, ApJ, 459, 701

Han, Z., \& Webbink, R. F. 1999, A\&A, 349, L17

Han, Z., Tout, C. A., \& Eggleton, P. P. 2000, MNRAS, 319, 215

Hjellming, M. S., \& Taam, R. E. 1993, ApJ, 370, 709

Iben, I., \& Tutukov, A. 1984, ApJS, 54, 335

Iben, I., \& Tutukov, A. 1985, ApJS, 58, 661

Iben, I., Ritossa, C., \& García-Berro, E. 1997, ApJ, 489, 772

José, J., Coc, A., \& Hernanz, M. 1999, ApJ, 520, 347

José, J., \& Hernanz, M. 1998, ApJ, 494, 680

Kaspi, V. M., Johnston, S., Bell, J. F., et al. 1994, ApJ, 423, L43

Langer, N., Deutschmann, A., Wellstein, A., \& Hoflich, P. 2000, A\&A, 362, 1041

Miyaji, S., Nomoto, K., Yokoi, K., \& Sugimoto, D. 1980, PASJ, 32,303

Nelson, C. A., \& Eggleton, P. P. 2001, ApJ, in press [astro-ph/0010269]

Nieuwenhuijzen, H., \& de Jager, C. 1990, A\&A, 231, 134

Nomoto, K. 1984, ApJ, 277, 791

Palacios, A., Leroy, F., Charbonnel, C., \& Forestini, M. 2000, in The Galactic Halo: from globular clusters to field stars, ed. A. Noels, P. Magain, D. Caro, et al., 67

Paczyński, B. 1971, ARA\&A, 9, 183

Ritossa, C., García-Berro, E., \& Iben, I. 1995, ApJ, 460, 489

Ritossa, C., García-Berro, E., \& Iben, I. 1999, ApJ, 515, 381

Saio, H., \& Nomoto, K. 1998, ApJ, 500, 388

Smalley, B., Smith, K. C., Wonnacott, D., \& Allen, C. S. 1996, MNRAS, 278, 688

Swartzman, E., Kovetz, A., \& Prialnik, D. 1994, MNRAS, 269, 323

Tauris, T. M., \& Dewi, J. D. M. 2001, A\&A, in press

Umeda, H., Nomoto, K., Yamaoka, H., \& Wanajo, S. 1999, ApJ, 513, 861

Van der Linden, T. J. 1987, A\&A, 178, 170

Webbink, S. 1984, ApJ, 277, 355

Weidemann, V. 2000, A\&A, 363, 647

Woosley, S. E., Weaver, T. A., \& Taam, R. E. 1980, in Texas Workshop on Type I Supernovae, ed. J. C. Wheeler (Austin: Univ. of Texas Press), 96

Whyte, C. A., \& Eggleton, P. P. 1980, MNRAS, 190, 801

Whyte, C. A., \& Eggleton, P. P. 1985, MNRAS, 214, 357 